\title{
Self-Organization of a Recurrent RBF Neural Network Using an Information-Oriented Algorithm
}

\author{
Hong-Gui Han ${ }^{\mathrm{a}, \mathrm{b}, *}$, Ya-Nan Guo ${ }^{\mathrm{a}, \mathrm{b}}$, Jun-Fei Qiao, \\ ${ }^{a}$ College of Automation, Faculty of Information Technology, Beijing University of Technology, Beijing, 100124, China. \\ ${ }^{\mathrm{b}}$ Beijing Key Laboratory of Computational Intelligence and Intelligent System, Beijing, 100124, China. \\ E-mail address: Rechardhan@sina.com (H. G. Han), guoyanan@emails.bjut.edu.cn (Y. N. Guo) and isibox@ sina.com (J. F. Qiao). \\ * Corresponding author. Phone +861067391631 Fax +861067391631
}

\begin{abstract}
This paper investigates how to construct a recurrent radial basis function neural network (RRBFNN) by an information-oriented algorithm (IOA) and how to adjust the parameters by a gradient algorithm simultaneously. In this IOA-based RRBFNN (IOA-RRBFNN), the proposed IOA is used to calculate the information processing strength (IPS) of hidden neurons, such that the independent component contributions between the hidden neurons and output neurons can be extracted. Then, a novel self-organizing strategy is proposed to optimize the structure of RRBFNN based on the input IPS and output IPS of hidden neurons. Meanwhile, a gradient algorithm is developed to update the parameters of IOA-RRBFNN. The proposed IOA-RRBFNN can be used to organize the network structure and adjust the parameters to improve its performance. Finally, several examples are presented to illustrate the effectiveness of IOA-RRBFNN. The results demonstrate that the proposed IOA-RRBFNN is more competitive in solving the nonlinear system modeling problems compared with some existing methods.
\end{abstract}

Index Terms - Information-oriented algorithm; recurrent radial basis function neural network; information processing strength; component contributions.

\section{INTRODUCTION}

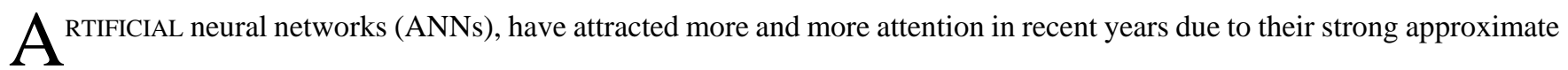
capacity [1]-[2]. Compared with the traditional methods, ANNs can be used to effectively approximate the nonlinear relationships between the input and output variables, and thus ANNs own great success in many kinds of subject areas and engineering applications, such as nonlinear systems modeling, intelligent adaptive control, pattern recognition, and so on [3]-[4]. In general, the structures of ANNs can be classified into two types: feedforward neural networks and recurrent neural networks [5]-[6]. Among different types of feedforward neural networks, the radial basis function neural networks (RBFNNs) have received considerable attention since they can be used to find solutions for nonlinear problems [7]-[8]. However, one of the main drawbacks of RBFNNs is that they are essentially static input-to-output maps and their ability of representing nonlinear systems is limited [9]-[11]. On the other hand, recurrent neural networks are capable of providing long-range predictions even in the presence of measurements noise [12]-[13]. In [14], the recurrent radial basis function neural network 
(RRBFNN) has been discussed, which has the ability of capturing various plant nonlinearities. It is noted that both RBFNNs and RRBFNNs can be used to approximate any nonlinear functions if their structures are properly constructed. In this case, the network size is often increased with the number of the sample data and a very large network size may be caused, which has the problem of poor generalization and high computational expense. Thus hidden neurons selection and structure optimization are of particular significance [15]-[17].

For designing the structure of RBFNNs, Huang et al. proposed a sequential learning algorithm for RBFNNs, which is referred to as the growing and pruning RBF algorithm (GAP-RBF) [18]. The original design of GAP-RBF was improved to produce a more advanced model known as the GGAP-RBF algorithm [19]. Both the GAP-RBF and GGAP-RBF algorithms use a pruning and growing strategy based on the "significance" of the hidden neurons. However, these two algorithms require a complete set of training samples in the training process. However, it is not usually possible for designers to have $a$ priori knowledge of all the training samples prior to the implementation [20]. Vukovi et al. exploited the concept of hidden neurons' significance and a growing and pruning algorithm is given in learning process [21]. Lee et al. also developed a growing and pruning technique based on the concept of hidden neurons' significance [22]. This proposed method can not only eliminate the influence of noise, but also dynamically adjust the number of hidden neurons to approach an appropriate size of RBFNN. However, the significance of hidden neurons is based on the average error of all the sequential input data which is suitable for offline training process [23]. To solve the problem, the methods based on evolutionary algorithm (EA) have been used to change the number of hidden neurons [24]-[25]. For example, Wu et al. introduced an effective hybrid optimization strategy, which incorporates the adaptive optimization of particle swarm optimization (PSO) into genetic algorithm (GA), such that the number of hidden neurons can be determined in [26]. Chen et al. combined the multi-innovation recursive least square (MRLS) and quantum particle swarm optimization (QPSO) algorithms together to adjust the parameters and network structure simultaneously [27]. In fact EAs has the great advantage in obtaining the global searching [28]-[29]. Furthermore, some other self-organizing methods (such as sensitivity analysis algorithm [30], visual cortex algorithm [31], spiking-based algorithm [32], and so on [33]-[35]) were used to optimize the structure of RBFNN. It is noted that in most existing self-organizing methods, only the structures of RBFNNs are discussed in the learning process.

In fact, the RBFNNs generated through these above self-organizing methods are with the feedforward type. The structure design of RRBFNNs is more complicated than that of RBFNNs due to the recurrent connections [36]-[37]. In order to obtain the suitable structures of RRBFNN, Subrahmanya et al. proposed a constructive method for structure and parameters training of RNNs simultaneously based on the PSO and covariance matrix adaptation strategy (PSOCMA-RNN) [38]. An Elman-based self-organizing RBF neural network (ESRNN) was designed through the simultaneous structure and parameters learning by the 
Mahalanobis distance approach in [39]. With both PSOCMA-RNN and ESRNN algorithms, the network size is increased while the redundant hidden neurons are not removed, so the network size may be too large in some applications. To prune the redundant hidden neurons, Zheng et al. introduced an interplay of spike-timing dependent plasticity and different homeostatic mechanisms for designing a class of recurrent neural networks [40]. Leung et al. proposed a local extended Kalman filtering training approach for pruning the hidden neurons of recurrent neural networks [41]. And a recursive Bayesian Levenberg-Marquardt algorithm, which can evaluate the significance of hidden neurons, was proposed to organize the recurrent neural networks in [42]. Although these recurrent neural networks with pruning methods [40]-[42] bring several benefits such as little redundancy of connections or neurons, the computational cost is high since the majority of the training time is spent on networks which have larger sizes than necessary. Recently, the combination of growing and pruning methods has become popular, which can effectively address some drawbacks of the individual growing or pruning algorithms, Based on the firing strength and the significance of each hidden neuron, Hsu et al. proposed a dynamic recurrent neural network which can generate or prune the hidden neurons online for achieving the optimal network structure [43]. The results show that this dynamic recurrent neural network can improve the generalization performance. El-Sousy developed a recurrent self-evolving fuzzy neural network (RRSEFNN), which can adjust the structure and parameter learning simultaneously [44]. The simulation results confirm that the proposed RRSEFNN obtains robust performance regardless of load disturbances. With these two algorithms [43]-[44], however, many constraint conditions should be assumed in the learning process. Wang et al. proposed an optimization algorithm for an Elman-type recurrent neural network, which combines the advantages of the discrete PSO algorithm and the improved PSO algorithm together [45]. The results show that this self-organizing recurrent neural network can obtain a low architectural complexity and good generalization performance. Other related results can be found in [46]-[47]. However, how to design an effective method for recurrent neural networks with the efficient performance is still a challenge [48]-[51].

Motivated by the above analysis, an information-oriented algorithm (IOA) is introduced to self-organize the structure of RRBFNN in this paper. This proposed IOA is a computational technique for identifying hidden independent sources from multivariate data, which is proposed to calculate the information processing strength (IPS) of hidden neurons. Then, a novel self-organizing strategy is proposed to optimize the network size of RRBFNN based on the input IPS and output IPS of hidden neurons. In general, the major contributions of this paper are summarized as follows: First, unlike the traditional approaches, the IOA-based self-organizing strategy uses not only the IPS of hidden neurons but also the information from the input data, which can effectively generate a network structure of RRBFNN. Second, the proposed self-organizing mechanism can add and prune the hidden neurons of RRBFNN without presetting any thresholds in the learning process. This feature is more useful in 
the applications compared with the existing methods [52]-[56]. Third, a gradient method is used to train the parameters of IOA-RRBFNN. Our proposed IOA-RRBFNN can determine the network size and adjust its parameters simultaneously, such that a network is obtained with a compact structure and high accuracy.

The rest of this paper is organized as follows. The next section briefly introduces the problem formulation. And the proposed IOA-RRBFNN is developed in details in Section III. In Section IV, several nonlinear function approximation and nonlinear systems modeling problems are applied to investigate the effectiveness of IOA-RRBFNN. The results demonstrate that the proposed IOA-RRBFNN has faster convergence speed and more accurate results than some existing methods. Finally, the conclusion is given in Section V.

\section{PROBLEM FORMULATION}

A three-layer RRBFNN is studied in this paper. The structure of the three-layer RRBFNN is shown in Fig. 1, which comprises the input layer, hidden layer and output layer. The neurons in the input layer receive the information from outside while the hidden neurons process the information from the combination of input layer and output layer. The Gaussian function is adopted as the activation function in the hidden layer due to its continuity and differentiability. The output neurons make the information integration from hidden layer. Furthermore, the prior output of the RBF neural network is recurrent to the hidden layer through a time delay. The mathematical description of RRBFNN is given below.

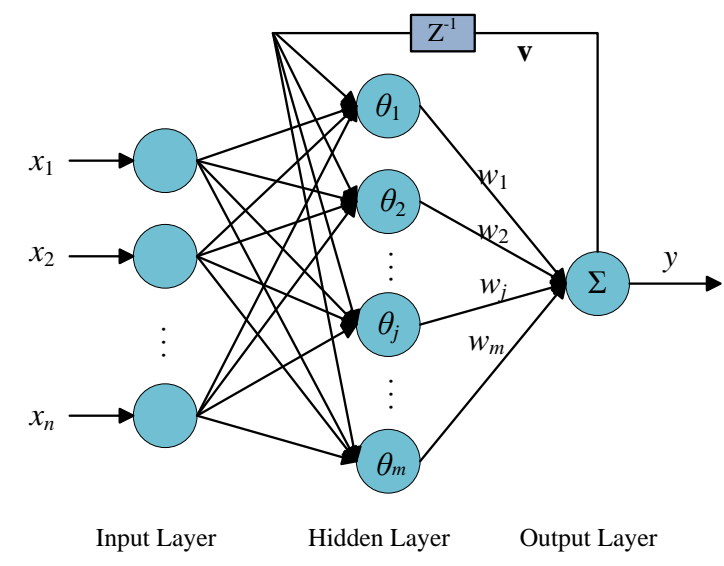

Fig. 1. The structure of RRBFNN

In this multi-input and single-output (MISO) RRBFNN, each layer is introduced as follows:

The input Layer: There are $n$ neurons which represent the input variables in this layer. The output values of each neuron are as follows:

$$
u_{i}(t)=x_{i}(t)
$$

where $u_{i}(t)$ is the $i$ th output value at time $t, i=1,2, \ldots, n$, and the input vector is $\mathbf{x}(t)=\left[x_{1}(t), x_{2}(t), \ldots, x_{n}(t)\right]$. 
The Hidden Layer: Each neuron in hidden layer connects with the input neurons and output neuron of RRBFNN. The outputs of hidden neurons are:

$$
\theta_{j}(t)=\mathrm{e}^{-\left\|\mathbf{h}_{j}(t)-\mathbf{c}_{j}(t)\right\| / 2 \sigma_{j}^{2}(t)}, j=1,2, \ldots, m,
$$

$\mathbf{c}_{j}(t)$ denotes the center vector of the $j$ th hidden neuron at time $t$, and $\mathbf{c}_{j}(t)=\left[c_{j 1}(t), c_{j 2}(t), \ldots, c_{j m+1}(t)\right]^{\mathrm{T}}, \| \mathbf{h}_{j}(t)$ - $\mathbf{c}_{j}(t) \|$ is the Euclidean distance between $\mathbf{h}_{j}(t)$ and $\mathbf{c}_{j}(t)$, and $\sigma_{j}(t)$ is the radius or width of the $j$ th hidden neuron at time $t, \mathbf{h}_{j}(t)$ is input vector of the $j$ th hidden neuron described by

$$
\mathbf{h}_{j}(t)=\left[u_{1}(t), u_{2}(t), \cdots u_{n}(t), v_{j}(t) \times y(t-1)\right]^{\mathrm{T}}
$$

$y(t-1)$ is the output value of output layer at time $t-1, v_{j}(t)$ denotes the connection weight from output layer to the $j$ th hidden neuron at time $t$, and $\mathbf{v}(t)=\left[v_{1}(t), v_{2}(t), \ldots, v_{m}(t)\right]^{\mathrm{T}}$.

The Output Layer: There is only one node in this layer, the output is:

$$
y(t)=f(\mathbf{w}(t), \boldsymbol{\theta}(t))=\sum_{j=1}^{m} w_{j}(t) \times \theta_{j}(t), j=1, \cdots, m
$$

where $\mathbf{w}(t)=\left[w_{1}(t), w_{2}(t), \ldots, w_{m}(t)\right]^{\mathrm{T}}$ is the connection weights between the hidden neurons and output neuron, $\boldsymbol{\theta}(t)=\left[\theta_{1}(t)\right.$ $\left.\theta_{2}(t), \ldots, \theta_{m}(t)\right]^{\mathrm{T}}$ is the output vector of the hidden layer, $y(t)$ represents the output of RRBFNN at time $t$.

Remark 1: It is clear that this RRBFNN has an internal feedback loop between the hidden layer and the output layer. It is expected that the internal feedback loop is able to enhance the capability of nonlinear systems modeling.

\section{DESIGN OF IOA-RRBFNN}

The basic idea of IOA-RRBFNN is to calculate the input and output IPS of hidden neurons by the IOA method, such that the network size is optimized. Meanwhile, a gradient decent algorithm is applied to adjust the parameters of IOA-RRBFNN. This section includes three parts: the IOA method for constructing the network size, the gradient learning approach for adjusting the parameters, and the main steps of IOA-RRBFNN.

\section{A. Information-oriented Algorithm (IOA)}

Without loss of generality, the relationship between the hidden neurons and the output neuron can be expressed as the generative equation:

$$
\begin{gathered}
\mathbf{y}(t)=\boldsymbol{\Phi}(t) \boldsymbol{\delta}(t), \\
\mathbf{y}(t)=[y(t-K+1), \cdots, y(t-1), y(t)]^{\mathrm{T}}, \\
\boldsymbol{\Phi}(t)=[\boldsymbol{\theta}(t-K+1), \cdots, \boldsymbol{\theta}(t-1), \boldsymbol{\theta}(t)]^{\mathrm{T}},
\end{gathered}
$$




$$
\boldsymbol{\delta}(t)=[\mathbf{w}(t-K+1), \cdots, \mathbf{w}(t-1), \mathbf{w}(t)],
$$

where $\mathbf{y}(t)=[y(t-K+1), \cdots, y(t-1), y(t)]^{\mathrm{T}}$, is output matrix of IOA-RRBFNN, $\mathbf{\Phi}(t)$ is output matrix of hidden layer, and $\boldsymbol{\delta}(t)$ is the weight matrix of hidden layer to output layer. It is noted that $K$ is the number of the samples, $y(t-K+1), \boldsymbol{\theta}(t-K+1)$ and $\mathbf{w}(t-K+1)$ are the output of IOA-RRBFNN, the output vector of the hidden layer and the weight vector at time $t-K+1$, respectively.

IOA is an independent component analysis algorithm, which can be used to calculate the statistical independence information of hidden neurons as well as extract the independent component contributions from the hidden neurons to output neurons. The expression is as follows

$$
\mathbf{Q}(t)=\mathbf{\Phi}(t) \mathbf{W}(t)
$$

where

$$
\mathbf{Q}(t)=\left[\begin{array}{cccc}
q_{1}(t-K+1) & q_{1}(t-K+2) & \cdots & q_{1}(t) \\
q_{2}(t-K+1) & q_{2}(t-K+2) & \cdots & q_{2}(t) \\
\vdots & \vdots & \cdots & \vdots \\
q_{m}(t-K+1) & q_{m}(t-K+2) & \cdots & q_{m}(t)
\end{array}\right],
$$

$\mathbf{Q}(t)=\left[\mathbf{q}_{1}(t), \ldots, \mathbf{q}_{J-1}(t), \mathbf{q}_{m}(t)\right]^{\mathrm{T}}$ is the independent contribution matrix, $\mathbf{q}_{j}(t)=\left[q_{j}(t-K+1), \ldots, q_{j}(t-1), q_{j}(t)\right]$ is the independent contribution of the $j$ th hidden neuron, $j=1, \ldots, m . \mathbf{W}(t)$ is a coefficients matrix which is given as

$$
\mathbf{W}(t)=\mathbf{D}^{-1}(t) \mathbf{\Phi}(t) \mathbf{B}(t) \mathbf{z}(t)
$$

where $\mathbf{D}(t), \mathbf{B}(t)$ and $\mathbf{z}(t)$ are the covariance matrix of $\mathbf{\Phi}(t)$, the whitening matrix of $\mathbf{y}(t)$ and the whitening transformation matrix of $\mathbf{y}(t)$, respectively. $\mathbf{D}(t), \mathbf{B}(t)$ and $\mathbf{z}(t)$ are given as

$$
\begin{gathered}
\mathbf{D}(t)=E\left\{\boldsymbol{\Phi}(t) \boldsymbol{\Phi}^{\mathrm{T}}(t)\right\}, \\
\mathbf{B}(t)=\boldsymbol{\Lambda}^{-1 / 2}(t) \mathbf{U}^{\mathrm{T}}(t), \\
\mathbf{z}(t)=\boldsymbol{\Lambda}^{-1 / 2}(t) \mathbf{U}^{\mathrm{T}}(t) \mathbf{y}(t),
\end{gathered}
$$

$\mathbf{U}(t)$ and $\mathbf{\Lambda}(t)$ are the eigenvector and eigenvalue matrices of $\mathbf{y}(t)$.

In this IOA, the values of covariance matrix $\mathbf{D}(t)$ is used to measure the correlation between $\mathbf{\Phi}(t)$ and $\mathbf{W}(t)$. Moreover, $\mathbf{D}^{-1}(t) \boldsymbol{\Phi}(t)$ is a de-correlation process for $\boldsymbol{\Phi}(t)$, which can represent the independence between the hidden neurons. And $\mathbf{B}(t) \mathbf{z}(t)$ can be used to reduce the correlation between hidden neurons and output layer. Based on the former analysis, the independent component contribution of hidden neurons is defined as 


$$
I_{j}(t)=\frac{\sum_{k=1}^{K} q_{j}(t-k+1)}{\sum_{k=1}^{K} \sum_{j=1}^{m} q_{j}(t-k+1)}, k=1, \cdots, K ; j=1, \cdots, m,
$$

where $I_{j}(t)$ is the independent component contribution of the $j$ th hidden neuron.

Remark 2: This IOA can be used to calculate the independence information between two hidden neurons and extract the independent component contributions from the hidden neurons to output neuron as shown in Eqs. (9)-(14). Based on the above discussion, this IOA introduces a more general framework by using the information of hidden neurons as shown in Eq. (15), which is more objective than other algorithms (see [18]-[45]).

\section{B. Self-Organizing Mechanism}

For this proposed IOA-RRBFNN, the hidden layer acts as a connecting bridge between input layer and output layer, which can map the incoming information to the output layer (see Fig. 2).

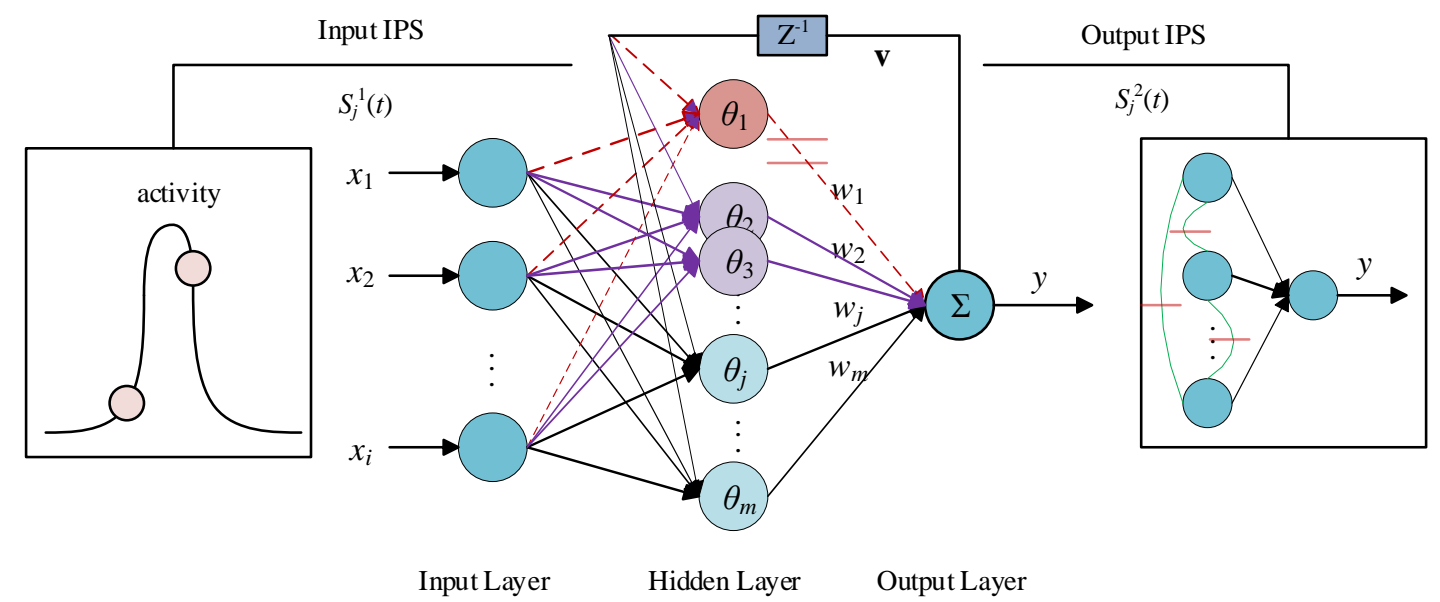

Fig. 2. Information process among the layers

In this IOA-RRBFNN, the IPSs are used to measure the competitive capacities of hidden neurons. The IPSs of hidden neurons are defined as

$$
\left\{\begin{array}{c}
S_{j}^{1}(t)=\frac{1}{K} \sum_{k=1}^{K} e^{-\left\|\mathbf{x}(t-k+1)-\mathbf{c}_{j}(t-k+1)\right\|} \\
S_{j}^{2}(t)=I_{j}(t)
\end{array}\right.
$$

where $S_{j}^{1}(t)$ and $S_{j}^{2}(t)$ are the input and output IPS of the $j$ th hidden neuron, respectively. This paper proposes these IPSs to assess the self-organizing mechanism for IOA-RRBFNN.

The proposed IOA-RRBFNN relies on the IPSs of hidden neurons. The self-organizing mechanism includes three rules used to design the structure of RRBFNN: the neuron splitting rule, the neuron deleting rule, and the neuron retaining rule. The cases of the self-organizing mechanism are given as follows: 


\section{Case 1: Neuron Splitting Rule}

$S_{j}^{1}(t)$ and $S_{j}^{2}(t)$ can be used to describe the information processing ability of hidden neurons. In fact, the larger $S_{j}^{1}(t)$ is, the input sample is closer to the center of the $j$ th hidden neuron. The $j$ th hidden neuron is active to this input sample. Meanwhile, based on Eqs. (9)-(15), the larger $S_{j}^{2}(t)$ is, the hidden neurons are more sensitive to the output neuron. If the IPSs of hidden neurons satisfy the condition:

$$
\left\{\begin{array}{l}
S_{j}^{1}(t)=\max \mathbf{S}^{1}(t) \\
S_{j}^{2}(t)=\max \mathbf{S}^{2}(t)
\end{array},\right.
$$

where $\mathbf{S}^{1}(t)=\left[S_{1}^{1}(t), \ldots, S_{m-1}^{1}(t), S_{m}^{1}(t)\right]$ and $\mathbf{S}^{2}(t)=\left[S_{1}^{2}(t), \ldots, S_{m-1}^{2}(t), S_{m}^{2}(t)\right]$ are the input IPS vector and the output IPS vector of hidden neurons, respectively. A new hidden neuron will be added into the network according to the $j$ th hidden neuron which owns both the maximum input IPS and output IPS:

$$
\left\{\begin{aligned}
\mathbf{c}_{\text {new }}(t) & =\alpha \mathbf{c}_{j}(t)+\beta \mathbf{h}_{j}(t), \\
\sigma_{\text {new }}(t) & =\alpha \sigma_{j}(t), \\
v_{\text {new }}(t) & =\alpha v_{j}(t), \\
w_{\text {new }}(t) & =w_{j}(t) \theta_{j}(t)\left(\theta_{\text {new }}(t)\right)^{-1},
\end{aligned}\right.
$$

where $\mathbf{c}_{j}(t)$ and $\sigma_{j}(t)$ represent the center and radius of pre-split $j$ th hidden neuron at time $t, \mathbf{c}_{\text {new }}(t)$ and $\sigma_{\text {new }}(t)$ stand for the center and radius of the new added hidden neuron, respectively, with $\alpha \in[0.95,1.05]$ and $\beta \in[0,0.1] ; v_{\text {newi }}(t)$ and $v_{j}(t)$ are the feedback weights of the new hidden neuron and the pre-split $j$ th hidden neuron at time $t$, respectively; $w_{\text {new }}(t)$ and $w_{j}(t)$ represent the connection weights of the new hidden neuron and the pre-split $j$ th hidden neuron at time $t$, respectively, $\theta_{\text {new }}(t)$ denotes the output of new added hidden neuron at time $t$.

\section{Case 2: Neuron Deleting Rule}

Based on the information processing ability of hidden neurons, the $i$ th hidden neuron will be deleted if the IPSs of hidden neurons satisfy the condition:

$$
\left\{\begin{array}{l}
S_{i}^{1}(t)=\min \mathbf{S}^{1}(t) \\
S_{i}^{2}(t)=\min \mathbf{S}^{2}(t)
\end{array}\right.
$$

The connection weights of the $i$ th hidden neuron will be updated according to

$$
w_{i^{\prime}}^{\prime}(t)=w_{i^{\prime}}(t)+w_{i}(t) \theta_{i}(t)\left(\theta_{i^{\prime}}(t)\right)^{-1},
$$

where $w_{i}^{\prime}(t)$ and $w_{i} \cdot(t)$ are the connection weights between the $i^{\prime}$ th hidden neuron and the output layer before and after the $i$ th hidden neuron is cut off, respectively; $\theta_{i^{\prime}}(t)$ and $\theta_{i}(t)$ are the outputs of the $i^{\prime}$ th and the $i$ th hidden neurons before the $i$ th hidden neuron is cut off. The center, radius and feedback weight of the $i$ 'th hidden neuron remain unchanged after the $i$ th hidden neuron is deleted. 


\section{Case 3: Neuron Retaining Rule}

When the input IPS and output IPS of hidden neurons are not the maximum information strength (such as Eq. (17)) or the minimum information strength (such as Eq. (19)), the hidden neurons will be retained and the structure of RRBFNN will not be changed.

With the self-organizing mechanism, the structure of RRBFNN can be automatically organized to improve the performance. Based on the aforementioned discussion, in contrast to some traditional algorithms, some properties of the self-organizing mechanism should be highlighted.

Remark 3: With the help of the input and output IPS, the active and inactive neurons can be identified. Then the splitting and deleting mechanisms can be used to find the suitable structure of RRBFNN. These observations lead to a new way of designing RRBFNN based on the information processing ability of hidden neurons, which contains the information from both the input layer and output layer. In this way, better judgement results can be obtained.

Remark 4: Based on the input and output IPS of hidden neurons, the self-organizing mechanism can add or prune the hidden neurons to organize the structure of RRBFNN in the learning process. The proposed self-organizing mechanism has two major advantages: 1) The neuron splitting mechanism and deleting mechanism can mimic the dynamics of hidden neurons to choose the right active and inactive neurons. The proposed method may also lead to the structure design of other kinds of recurrent neural networks. 2) Adjustments to the structure is performed without presetting any thresholds. This feature is useful in engineering applications.

\section{C.IOA-RRBFNN Training Process}

In the proposed IOA-RRBFNN, there are four types of parameters: the output weights $\mathbf{w}(t)$, the feedback connection weights $\mathbf{v}(t)$, the centers $\mathbf{c}(t)$ and the radii $\boldsymbol{\sigma}(t)=\left[\sigma_{1}(t), \sigma_{2}(t), \ldots, \sigma_{m}(t)\right]^{\mathrm{T}}$ of hidden neurons. These four types of parameters are updated as follows:

$$
\left\{\begin{array}{c}
\mathbf{c}(t+1)=\mathbf{c}(t)+\Delta \mathbf{c}(t) \\
\boldsymbol{\sigma}(t+1)=\boldsymbol{\sigma}(t)+\Delta \boldsymbol{\sigma}(t) \\
\mathbf{v}(t+1)=\mathbf{v}(t)+\Delta \mathbf{v}(t) \\
\mathbf{w}(t+1)=\mathbf{w}(t)+\Delta \mathbf{w}(t)
\end{array}\right.
$$

where

$$
\left\{\begin{array}{c}
\Delta \mathbf{c}(t)=-\eta_{1} \boldsymbol{\varphi}_{\mathbf{c}}(t) \mathbf{w}(t) e(t) \\
\Delta \boldsymbol{\sigma}(t)=-\eta_{2} \boldsymbol{\varphi}_{\mathbf{\sigma}}(t) \mathbf{w}(t) e(t) \\
\Delta \mathbf{v}(t)=-\eta_{3} \boldsymbol{\varphi}_{\mathbf{v}}(t) \mathbf{w}(t) e(t) \\
\Delta \mathbf{w}(t)=-\eta_{4} \boldsymbol{\varphi}_{\mathbf{w}}(t) e(t)
\end{array}\right.
$$




$$
\left\{\begin{array}{c}
\boldsymbol{\varphi}_{\mathbf{c}}(t)=\partial \boldsymbol{\theta}(t) / \partial \mathbf{c}(t) \\
\boldsymbol{\varphi}_{\mathbf{\sigma}}(t)=\partial \boldsymbol{\theta}(t) / \partial \boldsymbol{\sigma}(t) \\
\boldsymbol{\varphi}_{\mathbf{v}}(t)=\partial \boldsymbol{\theta}(t) / \partial \mathbf{v}(t) \\
\boldsymbol{\varphi}_{\mathbf{w}}(t)=\boldsymbol{\theta}(t)-\boldsymbol{\varphi}_{\mathbf{c}}(t) \mathbf{c}(t)-\boldsymbol{\varphi}_{\boldsymbol{\sigma}}(t) \boldsymbol{\sigma}(t)-\boldsymbol{\varphi}_{\mathbf{v}}(t) \mathbf{v}(t)
\end{array}\right.
$$

$\eta_{1}, \eta_{2}, \eta_{3}$, and $\eta_{4}$ are the positive learning rates, $e(t)$ is the current error of the neural network at time $t$ with $e(t)=y(t)-y_{d}(t), y_{d}(t)$ is the desired output. The partial derivative of the network output with respect to the centers of hidden neurons is given as

$$
\begin{aligned}
& \boldsymbol{\varphi}_{\mathbf{c}}(t)=\left[\begin{array}{cccc}
\boldsymbol{\varphi}_{c_{1}}(t) & 0 & \cdots & 0 \\
0 & \boldsymbol{\varphi}_{c_{2}}(t) & \cdots & 0 \\
\vdots & \vdots & \ddots & 0 \\
0 & 0 & \cdots & \boldsymbol{\varphi}_{c_{m}}(t)
\end{array}\right]^{\mathrm{T}} \\
& \boldsymbol{\varphi}_{c_{j}}(t)=\left[\varphi_{c_{j 1}}(t), \varphi_{c_{j 2}}(t), \cdots, \varphi_{c_{j n}}(t), \varphi_{c_{j(n+1)}}(t)\right]^{\mathrm{T}}, \\
& \varphi_{c_{j i}}(t)=\left\{\begin{array}{cc}
\theta_{j}(t)\left(x_{i}(t)-c_{j i}(t)\right) / \sigma_{j}^{2}(t), & i=1,2, \cdots, n \\
\theta_{j}(t)\left(v_{j}(t) y(t)-c_{j i}(t)\right) / \sigma_{j}^{2}(t), & i=n+1
\end{array}\right.
\end{aligned}
$$

the partial derivative of the network output with respect to the radii of hidden neurons is

$$
\begin{gathered}
\boldsymbol{\varphi}_{\sigma}(t)=\left[\begin{array}{cccc}
\varphi_{\sigma_{1}}(t) & 0 & \cdots & 0 \\
0 & \varphi_{\sigma_{2}}(t) & \cdots & 0 \\
\vdots & \vdots & \ddots & 0 \\
0 & 0 & \cdots & \varphi_{\sigma_{m}}(t)
\end{array}\right]^{\mathrm{T}} \\
\varphi_{\sigma_{j}}(t)=\theta_{j}(t)\left\|\mathbf{h}_{j}(t)-\mathbf{c}_{j}(t)\right\| / \sigma_{j}^{3}(t),
\end{gathered}
$$

the partial derivative of the network output with respect to the feedback connection weights is denoted as

$$
\begin{gathered}
\varphi_{\mathbf{v}}(t)=\left[\begin{array}{cccc}
\varphi_{v_{1}}(t) & 0 & \cdots & 0 \\
0 & \varphi_{v_{2}}(t) & \cdots & 0 \\
\vdots & \vdots & \ddots & 0 \\
0 & 0 & \cdots & \varphi_{v_{m}}(t)
\end{array}\right]^{\mathrm{T}} \\
\varphi_{v_{j}}(t)=-\theta_{j}(t) y(t)\left(v_{j}(t) y(t)-c_{j(n+1)}(t)\right) / \sigma_{j}^{2}(t),
\end{gathered}
$$

and the partial derivative of the network output with respect to the output weights is

$$
\begin{gathered}
\varphi_{\mathbf{w}}(t)=\left[\varphi_{w_{1}}(t), \varphi_{w_{2}}(t), \cdots, \varphi_{w_{m}}(t)\right]^{\mathrm{T}}, \\
\varphi_{w_{j}}(t)=\theta_{j}(t)-\varphi_{\mathbf{c}_{j}}^{\mathrm{T}}(t) \mathbf{c}_{j}(t)-\varphi_{\sigma_{j}}(t) \sigma_{j}(t)-\varphi_{v_{j}}(t) v_{j}(t),
\end{gathered}
$$


where $j=1,2, \ldots, m$. In the proposed IOA-RRBFNN, the self-organizing mechanism is used to tune the network structure.

Meanwhile, the gradient parameter adaptation strategy is applied to tune the parameters. The main procedures of training process of IOA-RRBFNN can be summarized in Table 1.

TABLE 1. The DetAILS OF IOA-RRBFNN TRAINING PROCESS

For IOA-RRBFNN, initialize the parameters: the output weights $\mathbf{w}$, the feedback connection weights $\mathbf{v}$, the centers $\mathbf{c}$ and the radii $\boldsymbol{\sigma}$.

For sample $\mathbf{x}(t)$

\section{\% Parameters learning process}

For all output weights, feedback connection weights, centers and radii of hidden neurons

End Update $\mathbf{w}, \mathbf{v}, \mathbf{c}$ and $\boldsymbol{\sigma}$;

$\%$ Eqs.(21)-(32)

\section{\%Structure self-organizing mechanisms}

For all hidden neurons

Calculate $S_{j}^{1}(t)$ and $S_{j}^{2}(t)$ of all hidden neurons;

If the input IPS and output IPS of the $j$ th hidden neuron satisfy (17)

Split the $j$ th hidden neuron and insert new hidden neurons, obtain the output weights, feedback weights, centers and the radii of new hidden neurons; If the input IPS and output IPS of the $j$ th hidden neuron satisfy (19)

Delete the $j$ th hidden neuron and update the output weights, feedback weights, centers and the radii of the retained hidden neurons; Eq.(20) End

End

Remark 5: It is noted that the training of the proposed IOA-RRBFNN contains two parts: structure organizing and parameters adjusting phases. Thus, the proposed IOA-RRBFNN has some good generalization properties.

\section{Simulation Studies}

In this section, five examples are used to verify the performance of IOA-RRBFNN: the nonlinear dynamic Mexican hat function, the Lorenz time series prediction, the Henon chaotic series prediction, the Box-Jenkins time series prediction and the total phosphorus prediction in wastewater treatment process (WWTP). And the results are compared with those in some other existing literatures. For simplicity, the initial parameters of the IOA-RRBFNN in these examples are the same: $\lambda=0.5, \sigma=0.69$, $c=0.09, w=0.05, v=0.9$. All the simulations were programmed with Matlab version 2010, and were run on a PC with a clock speed 2.6 GHz and 4 GB RAM, under a Microsoft Windows 8.0 environment.

The performances of the network are measured by the root-mean-squared error (RMSE). At time $t$, the RMSE is defined as:

$$
E(t)=\sqrt{\frac{1}{2 t} \sum_{p=1}^{t}\left(y_{d}(p)-y(p)\right)^{2}},
$$

where $y(p)$ and $y_{d}(p)$ are the network output and the desired output, respectively. 


\section{A. Mexican Hat Function}

In this example, the proposed IOA-RRBFNN is applied to approximate the following dynamic Mexican hat function, which has been used to demonstrate the performance of other neural networks:

$$
f\left(x_{1}, x_{2}\right)=\frac{\sin \left(\sqrt{x_{1}^{2}+x_{2}^{2}}\right)}{\sqrt{x_{1}^{2}+x_{2}^{2}}},
$$

where $x_{1}, x_{2} \in[-2 \pi, 2 \pi]$, there are two inputs and one output in this IOA-RRBFNN. A set of 600 points randomly generated were chosen as the training samples, and 400 data points randomly generated were used as the testing samples. The initial number of hidden neurons was 2. The pre-set training RMSE was 0.01. The experiment results are presented in Figs. 3-6.

The number of hidden neurons in training process is displayed in Fig. 3. The RMSE values during the training process are shown in Fig. 4. Meanwhile, the approximating results and approximating errors are shown in Figs. 5-6. Based on the results given in Figs. 3-6, the proposed approach gives a good approximation of the dynamic Mexican hat function. In order to evaluate the performance of IOA-RRBFNN, its results were compared with those of six other neural networks: the RBF neural network (RBFNN), the recurrent RBF neural network (RRBFNN), the IOA-RBF neural network (IOA-RBFNN), the ART-RBF neural network (ART-RBFNN) [33], the DFNN [23], and the GDFNN [56]. Notably, the IOA-RBFNN is the RBFNN with IOA. All the algorithms have the same training data sets as well as testing partitions.

Table 2 shows the comparison in terms of the final number of hidden neurons, the testing RMSE, and the CPU times. It can be seen that the proposed IOA-RRBFNN obtains the most compact structure ( 9 hidden neurons), the best testing RMSE (0.0213) and the shortest CPU time (7.43s) in this example. The simulation results mean that the proposed IOA-RRBFNN can approximate the dynamic Mexican hat function with better approximation and generalization performance compared with other neural networks.

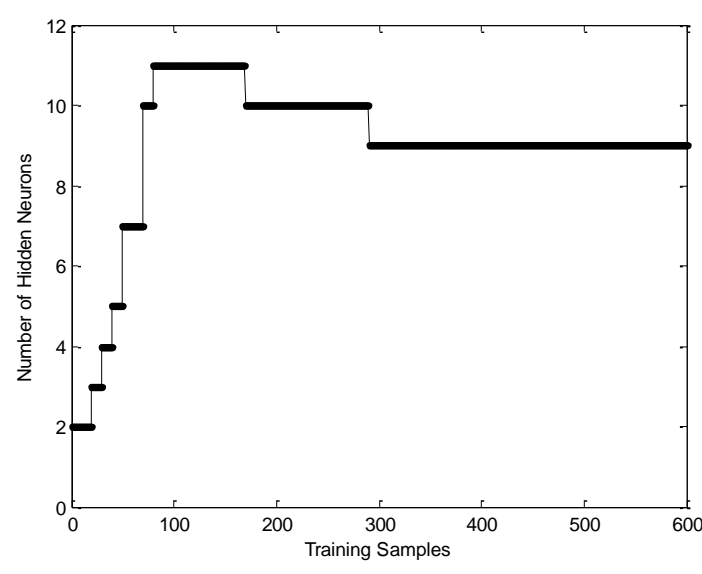

Fig. 3. The number of hidden neurons in the training process

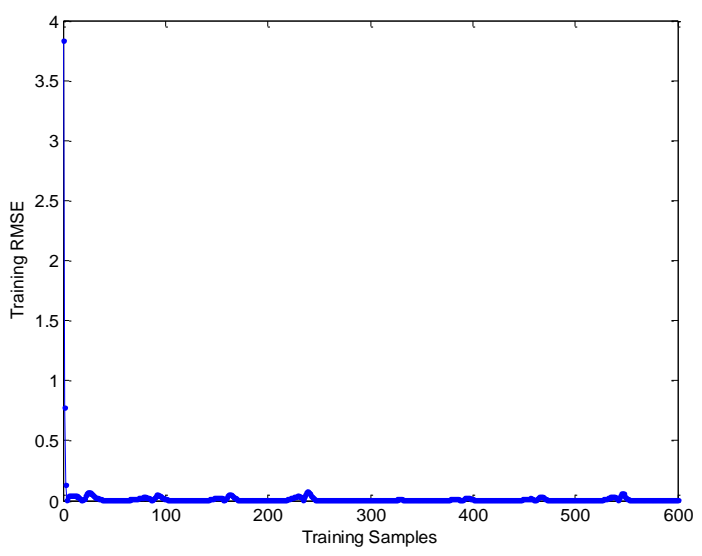

Fig. 4. The RMSE values in the training process 


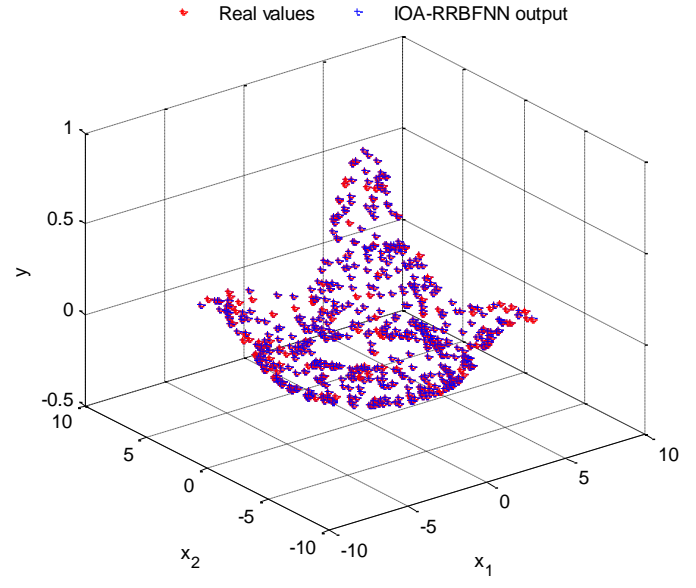

Fig. 5. The approximating results by IOA-RRBFNN

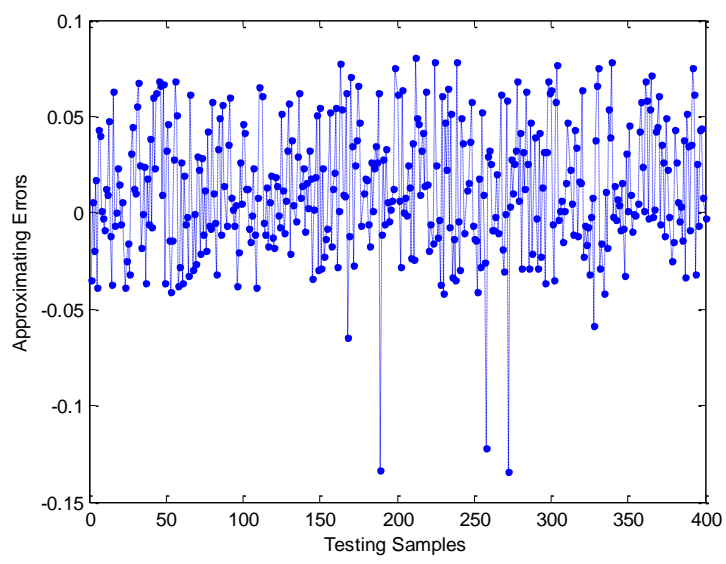

Fig. 6. The approximating errors versus testing samples

TABLE 2. EXPERIMENTAL RESUltS OF MEXICAN HAT FUNCTION APPROXIMATION

\begin{tabular}{ccccc}
\hline Algorithms & $\begin{array}{c}\text { Hidden } \\
\text { Neurons }\end{array}$ & $\begin{array}{c}\text { Training } \\
\text { RMSE }\end{array}$ & $\begin{array}{c}\text { Testing } \\
\text { RMSE }\end{array}$ & CPU Time $(s)$ \\
\hline IOA-RRBFNN & $\mathbf{9}$ & $\mathbf{0 . 0 0 9 2}$ & $\mathbf{0 . 0 2 1 3}$ & $\mathbf{7 . 4 3}$ \\
\hline RBFNN & 35 & 0.0568 & 0.1347 & 15.69 \\
RRBFNN & 30 & 0.0289 & 0.0933 & 20.81 \\
IOA-RBFNN & 15 & 0.0103 & 0.0318 & 8.02 \\
ART-RBFNN [33] & $33^{*}$ & $0.0547^{*}$ & $0.0556^{*}$ & $13.76^{*}$ \\
DFNN [23] & $12^{*}$ & $0.0191^{*}$ & $0.0348^{*}$ & $45.63^{*}$ \\
GDFNN [56] & $11^{*}$ & $0.0108^{*}$ & $0.0279^{*}$ & $43.21^{*}$ \\
\hline
\end{tabular}

* The results are not listed in the original papers.

Remark 6: In all five examples, the final network structure is the steadiest over 50 trials of simulations. Also, each algorithm was run for 50 times and the presented performance is the average value of 50 trials.

\section{B. Lorenz Time Series Prediction}

Lorenz system has been recognized as one of the benchmark problems for assessing the performance of learning algorithms.

The Lorenz time series is generated by the following discrete equation:

$$
\left\{\begin{array}{c}
\frac{d x(t)}{d t}=a y(t)-a x(t) \\
\frac{d y(t)}{d t}=c x(t)-x(t) z(t)-y(t), \\
\frac{d z(t)}{d t}=x(t) y(t)-b z(t)
\end{array}\right.
$$

where $a=10, b=8 / 3$, and $c=28$ are the system parameters. In this example, the fourth-order Runge-Kutta approach with a step size of 0.01 is adopted to generate the Lorenz samples, and only the $Y$-dimension samples $y(t)$ are used for the time series prediction. For the 1500 data samples generated for $y(t)$, the first 1000 samples were taken as training data, and the last 500 samples were used to check the proposed model. The initial number of hidden neurons was 10. The pre-set training RMSE was 0.01. The results are given in Figs. 7-10 and the details of compassion are shown in Table 3. 


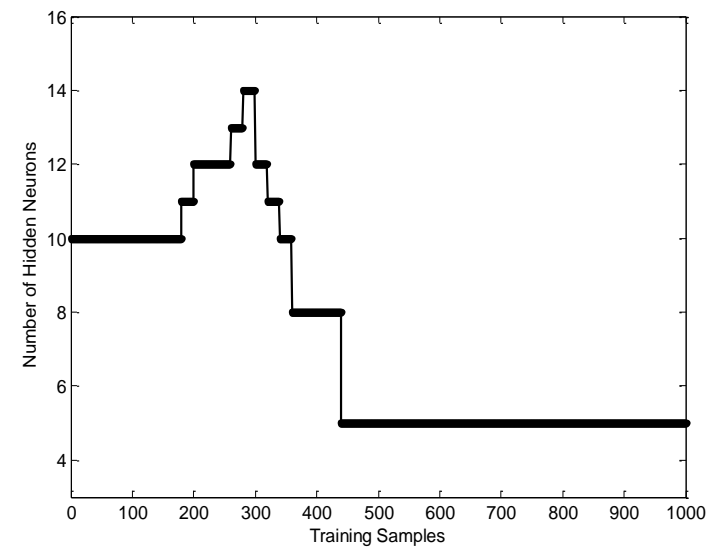

Fig. 7. The number of hidden neurons in the training process

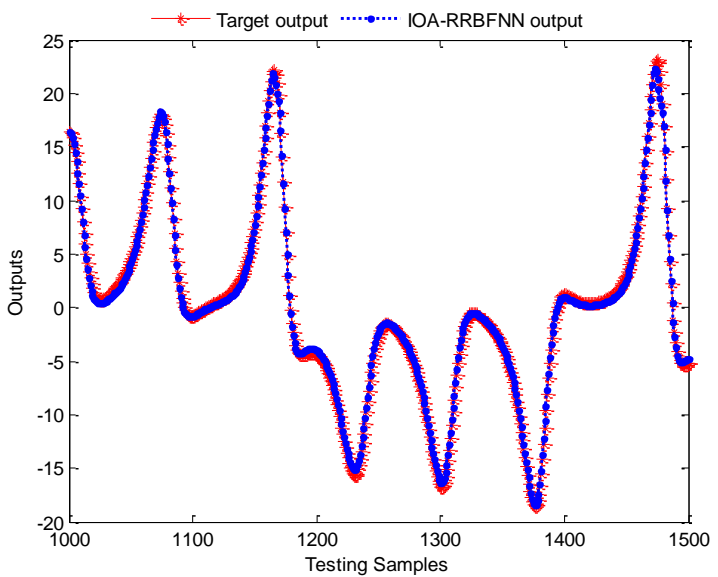

Fig. 9. The predicting results by IOA-RRBFNN

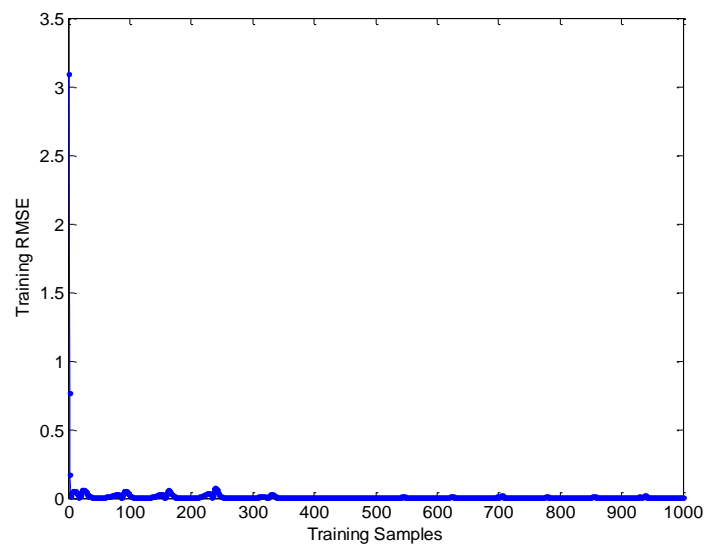

Fig. 8. The RMSE values in the training process

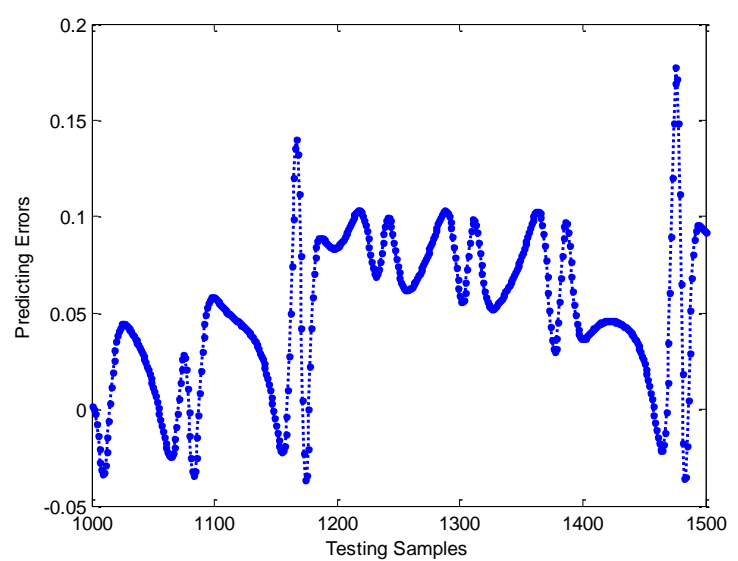

Fig. 10. The predicting errors versus testing samples

Fig. 7 shows the number of hidden neurons in training process. And the RMSE values during the training process are displayed in Fig. 8. The effect curve during testing process while output of the actual output compared to the desired output are given in Figs. 9-10. Meanwhile, IOA-RRBFNN were compared with the feedforward type network (the RBFNN, the resource allocation network with EKF (RANEKF) [53], and the modified differential evolution of RBF neural network (MDE-RBFNN) [34]), the recurrent type network (the recurrent neural network via backpropagation through time (RNN-BPTT) [42], the recurrent neural network via recursive Bayesian Levenberg-Marquardt (RBLM-RNN) [42], the recurrent neural network via extended Kalman filter (RNN-EKF) [42], the recurrent neural network via real-time recurrent learning (RNN-RTRL) [42], the autoregressive moving average artificial neural network (ARMANN) [54], the recursive least squares echo state network (RLS-ESN) [48]), and the self-organizing type network (the IOA-RBFNN, the recurrent self-evolving fuzzy neural network (RSEFNN) [28], and the pseudo-Gaussian RBF neural network (PG-RBFNN) [35]). In such comparisons, the same training data sets and testing partitions are adopted.

It can be seen from table 3 that the number of hidden neurons of IOA-RRBFNN is 5 which illustrates that the proposed IOA-RRBFNN owns a more compact structure of the neural network compared with other methods. In addition to, the testing 
RMSE of the network is 0.0274 , which is also far less than the others (except the RSEFNN [28]). The CPU time of the network is 9.71, which is also shorter than the others (except the IOA-RBFNN). Hence it can be observed that the proposed IOA-RRBFNN has more compact structure, lower testing RMSE and shorter CPU time.

TABle 3. EXPERIMENTAL RESUlts OF LORENZ TIME SERIES PREDICTION

\begin{tabular}{|c|c|c|c|c|c|}
\hline \multicolumn{2}{|c|}{ Algorithms } & $\begin{array}{c}\text { Hidden } \\
\text { Neurons }\end{array}$ & $\begin{array}{l}\text { Training } \\
\text { RMSE }\end{array}$ & $\begin{array}{c}\text { Testing } \\
\text { RMSE }\end{array}$ & CPU Time $(s)$ \\
\hline \multirow{4}{*}{ Feedforward type } & RBFNN & 10 & 0.0273 & 0.0985 & 26.33 \\
\hline & RANEKF [53] & 26 & 0.2260 & 0.2092 & 46.30 \\
\hline & MDE-RBFNN [34] & 10 & $0.0211 *$ & 0.1700 & $41.13^{*}$ \\
\hline & ARMANN [54] & $50 *$ & 0.0523 & 0.0876 & 53.02 \\
\hline \multirow{6}{*}{ Recurrent type } & RNN-BPTT [42] & 19 & 0.0277 & 0.0436 & 44.53 \\
\hline & RNN-RTRL [42] & 19 & 0.0229 & 0.0420 & 50.21 \\
\hline & RNN-EKF [42] & 19 & 0.0182 & 0.0352 & 62.98 \\
\hline & RBLM-RNN [42] & 16 & 0.0182 & 0.0304 & 59.74 \\
\hline & RRBFNN & 18 & 0.0177 & 0.0539 & 50.69 \\
\hline & RLS-ESN [48] & $300 *$ & 0.5033 & 0.0661 & $67.54 *$ \\
\hline \multirow{4}{*}{ Self-organizing type } & IOA-RRBFNN & 5 & 0.0158 & 0.0274 & 9.71 \\
\hline & IOA-RBFNN & 8 & 0.0175 & 0.0812 & 7.32 \\
\hline & RSEFNN [28] & 7 & 0.0131 & 0.0142 & $143.23 *$ \\
\hline & PG-RBFNN [35] & 6 & $0.0226 *$ & 0.0940 & $31.12 *$ \\
\hline
\end{tabular}

* The results are not listed in the original papers.

\section{Henon Chaotic Series Prediction}

In this example, the proposed IOA-RRBFNN is applied to predict the Henon chaotic system with one delay and two sensitive parameters generated by the following equation:

$$
y_{p}(t+1)=-p y_{p}^{2}(t)+b y_{p}(t-1)+1.0
$$

where $p=1.4$ and $b=0.3$ with a chaotic attractor. The initial state is $\left[y_{p}(1), y_{p}(0)\right]=[0.4,0.4] .1000$ data points were used as the training samples and the other 1000 data points were used as the testing samples. The pre-set training RMSE was 0.01 . The initial number of hidden neurons was 10 .

The number of hidden neurons and the RMSE values in training process are displayed in Figs. 11-12, respectively. The data in Figs. 13-14 show that the proposed IOA-RRBFNN can be used to predict the Henon chaotic system. Based on the results in Figs. 11-14, the proposed IOA-RRBFNN gives a good prediction of the Henon chaotic system. Moreover, to evaluate the performance of IOA-RRBFNN, the results were compared with those of the other methods: the RRBFNN, the recurrent FNN (RFNN) [49], the RLS-ESN [48], the wavelet recurrent FNN (WRFNN) [50], the TSK-type recurrent fuzzy network with supervised learning (TRFN-S) [36], the recurrent self-evolving FNN with local feedback (RSEFNN-LF) [28], the interactively recurrent self-evolving fuzzy neural network composed of a Takagi-Sugeno-Kang (IRSFNN-TSK) [29], the functional-link-based IRSFNN (IRSFNN-FuL) [29] and the IOA-RBFNN. 


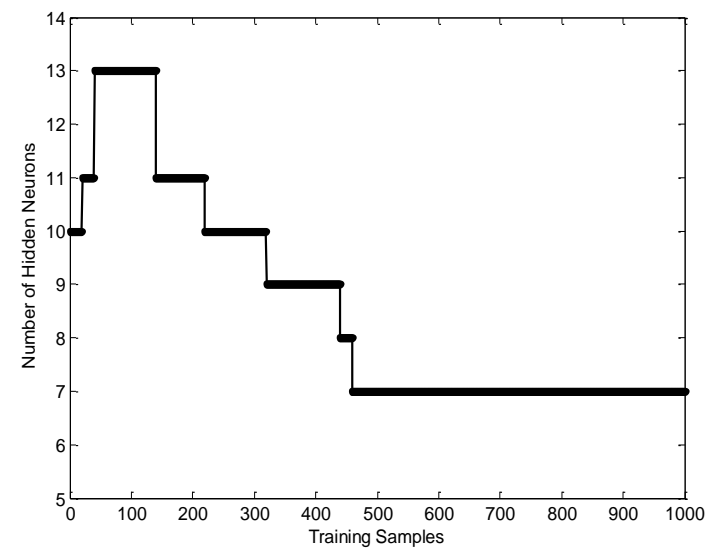

Fig. 11. The number of hidden neurons in the training process

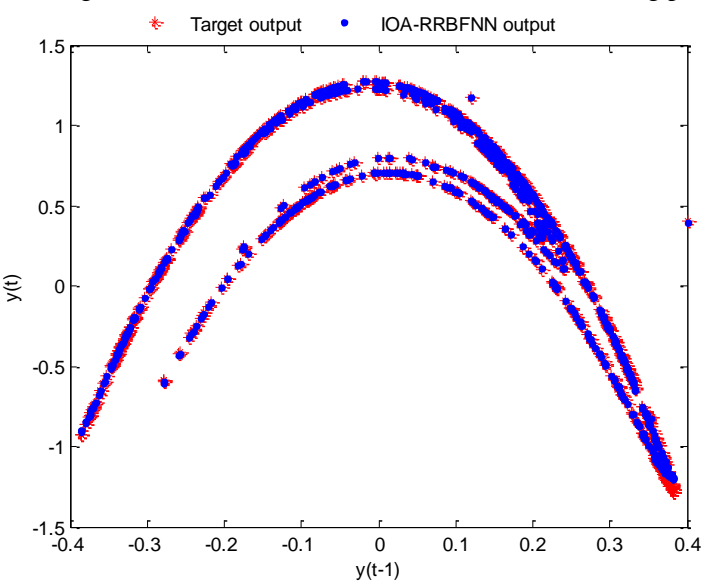

Fig. 13. The predicting results by IOA-RRBFNN

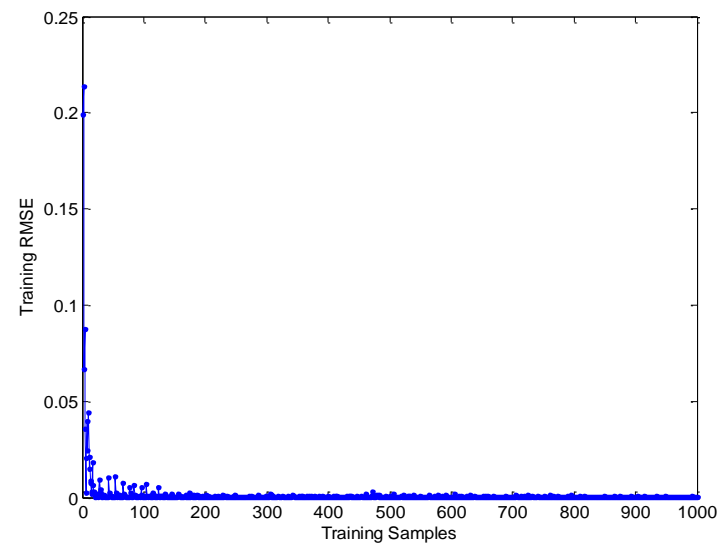

Fig. 12. RMSE values in the training process

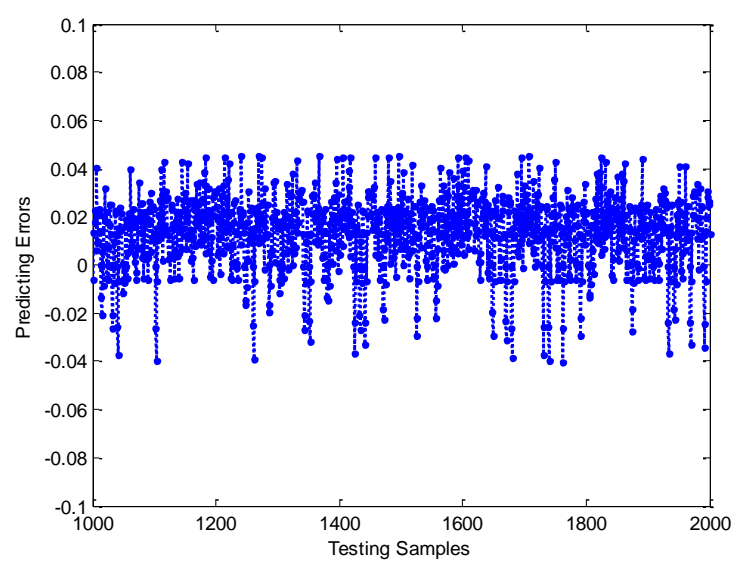

Fig. 14. The predicting errors versus testing samples

TABle 4. EXPerimental Results of HenON ChaOtic Series Prediction

\begin{tabular}{|c|c|c|c|c|c|}
\hline \multicolumn{2}{|c|}{ Algorithms } & $\begin{array}{c}\text { Hidden } \\
\text { Neurons } \\
\end{array}$ & $\begin{array}{c}\text { Training } \\
\text { RMSE } \\
\end{array}$ & $\begin{array}{l}\text { Testing } \\
\text { RMSE }\end{array}$ & CPU Time $(s)$ \\
\hline \multirow{5}{*}{ Recurrent type } & RRBFNN & 18 & 0.0180 & 0.0350 & 24.03 \\
\hline & RFNN [49] & 15 & 0.4630 & 0.4690 & 10.68 \\
\hline & RLS-ESN [48] & $300 *$ & 0.0141 & 0.0130 & $43.21 *$ \\
\hline & WRFNN [50] & 7 & 0.1910 & 0.1880 & 23.89 \\
\hline & TRFN-S [36] & 6 & 0.0280 & 0.0270 & $27.18^{*}$ \\
\hline \multirow{5}{*}{ Self-organizing type } & IOA-RRBFNN & 7 & 0.0116 & 0.0127 & 14.36 \\
\hline & RSEFNN-LF [28] & 9 & 0.0320 & 0.0230 & $33.31 *$ \\
\hline & IRSFNN-FuL [29] & 4 & 0.0170 & 0.0150 & $27.72 *$ \\
\hline & IRSFNN-TSK [29] & 3 & 0.0160 & 0.0140 & $26.16^{*}$ \\
\hline & IOA-RBFNN & 10 & 0.0220 & 0.0280 & 11.71 \\
\hline
\end{tabular}

* The results are not listed in the original papers.

The details of compassion are shown in Table 4 with respect to the following indices: the final number of hidden neurons, the CPU time, and the testing RMSE. It can be seen from table 4 that the number of hidden neurons of IOA-RRBFNN is 7 which is less than that of the other methods (except the TRFN-S [36], the IRSFNN-FuL [29] and the IRSFNN-TSK [29]). The testing RMSE of IOA-RRBFNN is the smallest among these different methods. Also, the proposed IOA-RRBFNN needs lest CPU 
time (14.36 s) among all methods (except the RFNN [49]). This example proves that the IOA-RRBFNN has better identification ability than some other existing neural networks.

\section{D.Box-Jenkins Times Series Prediction}

In this example, the Box-Jenkins time series, which describes the operation of a gas furnace process with a gas flow rate - $u(t)$ and a concentration of $\mathrm{CO}_{2}-y(t)$, is used to evaluate the IOA-RRBFNN performance. $u(t-4)$ and $y(t-1)$ are used as the inputs of IOA-RRBFNN to predict $y(t)$. A set of 296 data points is provided. 196 data points are used as training samples and the remained 100 data points are used as the testing samples. The initial number of hidden neurons was 3 . The pre-set training RMSE was 0.001.

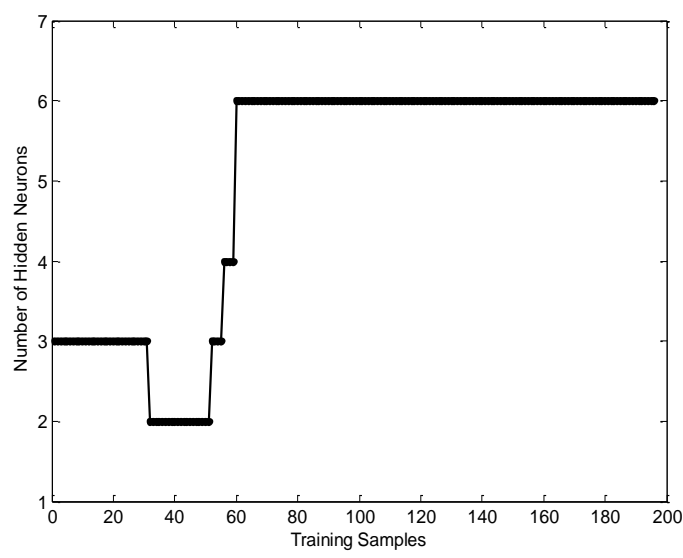

Fig. 15. The number of hidden neurons in the training process

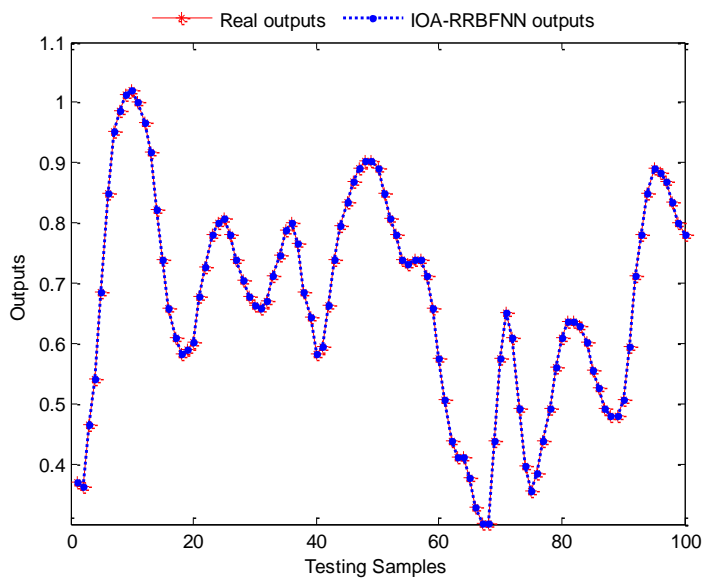

Fig. 17. The predicting results by IOA-RRBFNN

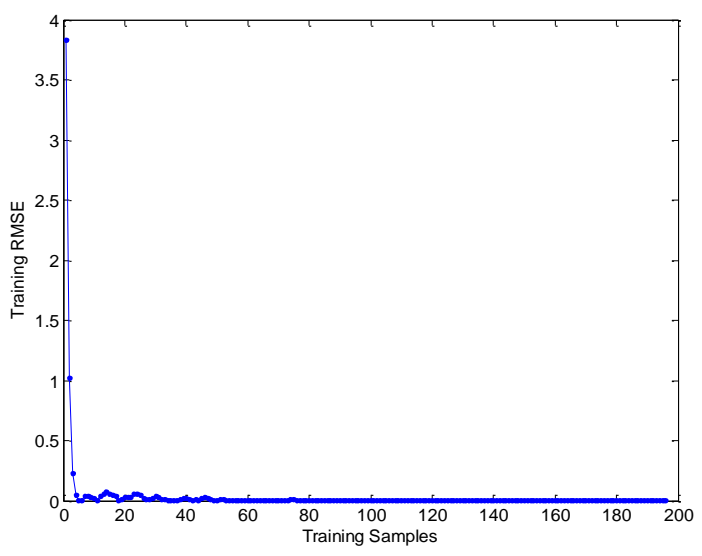

Fig. 16. The RMSE values in the training process

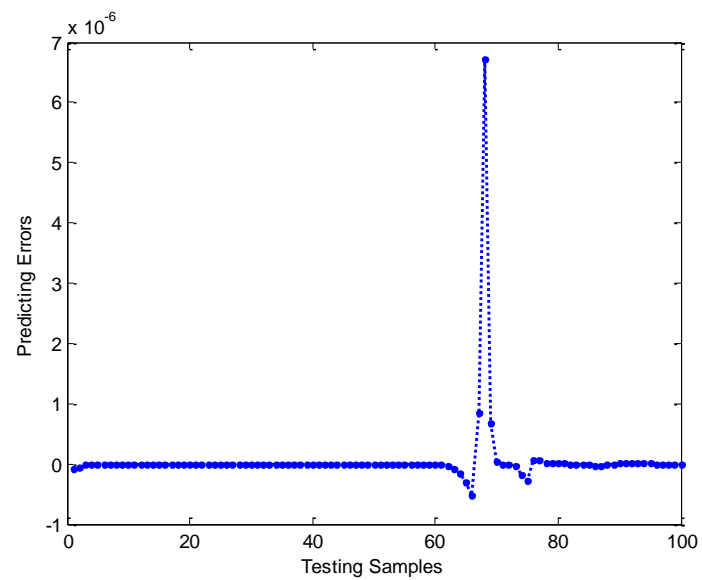

Fig. 18. The predicting errors versus testing samples

The predicting results for this Box-Jenkins time series system are displayed in Figs. 15-18, including the number of hidden neurons in training process, the RMSE values in training process and the predictions via the proposed IOA-RRBFNN algorithm. The graphs in Figs. 17-18 demonstrate that the proposed IOA-RRBFNN can predict this nonlinear system with a satisfactory accuracy and good generalization performance. In addition, the results are compared with those of the feedforward type network (the tree-based neural fuzzy inference system (TNFIS) [51], the Adaptive neuro-fuzzy inference systems (HyFIS) 
[52], the local linear wavelet neural network (LLWNN) with hybrid learning (LLWNN+hybrid) [55], the LLWNN with gradient learning (LLWNN+gradient) [55], and the fuzzy wavelet neural network (FWNN) [57]), the recurrent type network (the RRNN, the TRFN-S [36] and the recurrent adaptive neural fuzzy inference system (RANFIS) [52]), and the self-organizing type network (the RSEFNN-LF [28], the IRSFNN-TSK [29], the IRSFNN-FuL [29], and the IOA-RBFNN).

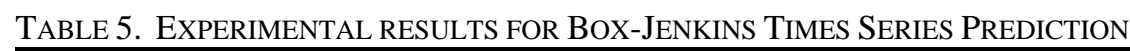

\begin{tabular}{clcccc}
\hline \multirow{2}{*}{ Algorithms } & $\begin{array}{c}\text { Hidden } \\
\text { Neurons }\end{array}$ & $\begin{array}{c}\text { Training } \\
\text { RMSE }\end{array}$ & $\begin{array}{c}\text { Testing } \\
\text { RMSE }\end{array}$ & CPU Time $(s)$ \\
\hline \multirow{5}{*}{ Feedforward type } & TNFIS [51] & 13 & 0.0245 & 0.0230 & $83.56^{*}$ \\
& HyFIS [52] & 15 & 0.0382 & 0.0588 & $78.09^{*}$ \\
& LLWNN+Hybrid [55] & 8 & 0.0109 & 0.0138 & 153.00 \\
& LLWNN+gradient [55] & 8 & 0.0158 & 0.0164 & 125.00 \\
& FWNN [57] & 9 & 0.0189 & 0.0279 & $101.12^{*}$ \\
\hline \multirow{5}{*}{ Recurrent type } & TRFN-S [36] & 5 & 0.0524 & 0.0482 & $32.09^{*}$ \\
& RRBFNN & 8 & 0.0052 & 0.0109 & $18.02^{*}$ \\
& RANFIS [52] & 20 & 0.0060 & 0.0193 & $19.89^{*}$ \\
\hline \multirow{5}{*}{ Self-organizing type } & IOA-RRBFNN & $\mathbf{6}$ & $\mathbf{0 . 0 0 1 1}$ & $\mathbf{0 . 0 0 1 7}$ & $\mathbf{9 . 2 1}^{*}$ \\
& RSEFNN-LF [28] & 7 & 0.0172 & 0.0344 & $23.21^{*}$ \\
& IRSFNN-TSK [29] & 5 & 0.0121 & 0.0297 & $26.29^{*}$ \\
& IRSFNN-FuL [29] & 3 & 0.0043 & 0.0097 & $27.54^{*}$ \\
& IOA-RBFNN & 13 & 0.0101 & 0.0291 & 8.07 \\
\hline
\end{tabular}

* The results are not listed in the original papers.

The details of the comparisons of this example are given in Table 5. It can be seen from Table 5 that the proposed IOA-RRBFNN needs least CPU time (except for the IOA-RBFNN). Also, the proposed IOA-RRBFNN obtains better testing RMSE (0.0017). Hence, the proposed IOA-RRBFNN is more suitable for this Box-Jenkins time series prediction than the other methods.

\section{E. Total Phosphorus Prediction}

Wastewater treatment process is one of the most effective methods to solve the problem of water pollution. The total phosphorus (TP) is an important parameter to evaluate the performance of wastewater treatment process. However, how to online measure the reliable values of TP is still a widespread problem in the operation of wastewater treatment process. In this experiment, the proposed IOA-RRBFNN is used to predict the effluent TP values. We select some easy-to-measure process variables as the input variables for IOA-RRBFNN, which include the total suspended solids (TSS), the temperature (T), the dissolved oxygen concentration (DO), the $\mathrm{pH}$, and the influent TP. The explanations of the process parameters are listed in Table 6.

In this example, the experience data were collected from 1/6/2014 to 1/5/2015 of a real WWTP in Beijing, China. After the abnormal data deletion, 145 data points were used as training data and 120 data points were used as testing data. And the former 60 sets data were obtained from sunny day, the latter 60 from rainy day. We intend to test the performance of proposed network 
by a changed scenarios. The initial number of hidden neurons is 4 . The error measures for TP are $0.1 \mathrm{mg} / \mathrm{L}$ confidence limits.

The experiment results are presented in Figs. 19-22.

TABLE 6. INPUTS AND OUTPUT VARIABLES USED IN THIS STUDY

\begin{tabular}{ccc}
\hline Variables & Explanation & Type of variables \\
\hline TSS $(\mathrm{mg} / \mathrm{L})$ & Total suspended solids & Input variable \\
$\mathrm{T}$ & Temperature & Input variable \\
$\mathrm{DO}(\mathrm{mg} / \mathrm{L})$ & Dissolved oxygen concentration & Input variable \\
$\mathrm{pH}$ & Acidity and basicity & Input variable \\
Influent TP $(\mathrm{mg} / \mathrm{L})$ & Influent total phosphorus & Input variable \\
Effluent TP $(\mathrm{mg} / \mathrm{L})$ & Effluent total phosphorus & Output variable \\
\hline
\end{tabular}

TABle 7. EXPERIMENTAL RESUlts of the TOtal PHOSPHORUS PREDICTION

\begin{tabular}{cccc}
\hline Algorithms & Hidden Neurons & Testing RMSE & Prediction Accuracy $(\%)$ \\
\hline IOA-RRBFNN & $\mathbf{7}$ & $\mathbf{0 . 1 0 3 3}$ & $\mathbf{9 3 . 6}$ \\
\hline RBFNN & 10 & 0.4092 & 83.2 \\
RRBFNN & 8 & 0.2279 & 88.1 \\
IOA-RBFNN & 8 & 0.1806 & 90.5 \\
RSEFNN-LF [28] & $7 *$ & $0.1241^{*}$ & $92.0^{*}$ \\
TRFN-S [36] & $6 *$ & $0.2570^{*}$ & $87.6^{*}$ \\
IRSFNN-TSK [29] & $6 *$ & $0.1908^{*}$ & $89.7 *$ \\
IRSFNN-FuL [29] & $7 *$ & $0.1877^{*}$ & $90.3 *$ \\
RANFIS [52] & $20^{*}$ & $0.1179^{*}$ & $92.8^{*}$ \\
\hline
\end{tabular}

* The results are not listed in the original papers.

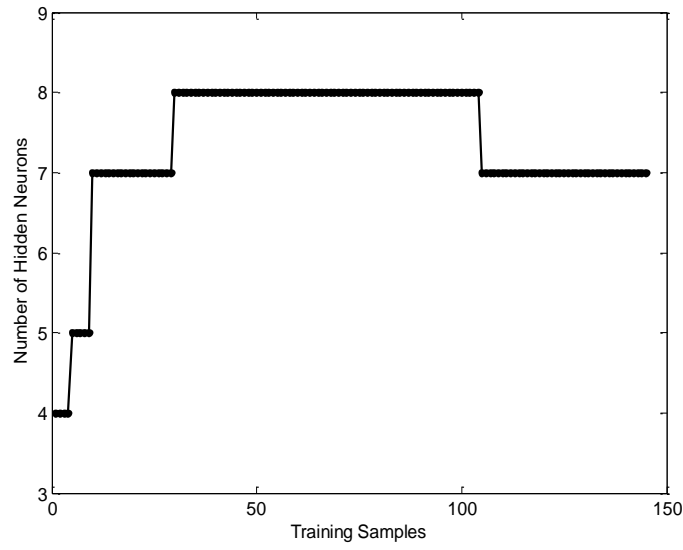

Fig. 19. The number of hidden neurons in the training process

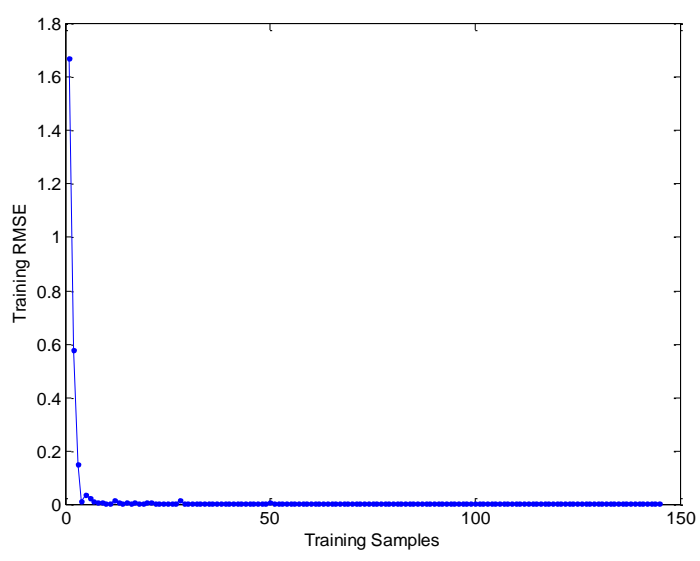

Fig. 20. The RMSE values in the training process

The number of hidden neurons and the RMSE values in training process are displayed in Figs. 19-20, respectively. The TP values by the IOA-RRBFNN method are depicted together with the real process output in Fig. 21 . In order to check the predicting ability of IOA-RRBFNN, the predicting errors of TP are shown in Fig. 22. To show the performance of IOA-RRBFNN, the predicting values are compared with those of the RBFNN, the RRBFNN, the ISO-RBFNN, the RSEFNN-LF [28], the TRFN-S [36], the IRSFNN-TSK [29], the IRSFNN-FuL [29], and the RANFIS [52]. For fair comparison, the optimal parameters of the models are the same as the initial papers. Performance is measured by the final 
number of hidden neurons, the testing RMSE and the predicting accuracy as defined in [44]. The details are presented in Table 7. As Table 7 indicates, a higher mean accuracy is achieved by the proposed IOA-RRBFNN (mean accuracy value is $93.6 \%$ ). Meanwhile, Table 7 illustrates that the mean accuracy by the proposed IOA-RRBFNN is higher than those by other methods, which can also show that this proposed IOA-RRBFNN may improve the chance to approximate the global optimization parameters in the learning process. Furthermore, the results demonstrate that the TP trends in the wastewater treatment process can be predicted with acceptable accuracy using the TSS, T, DO, pH, and influent TP data as input variables.

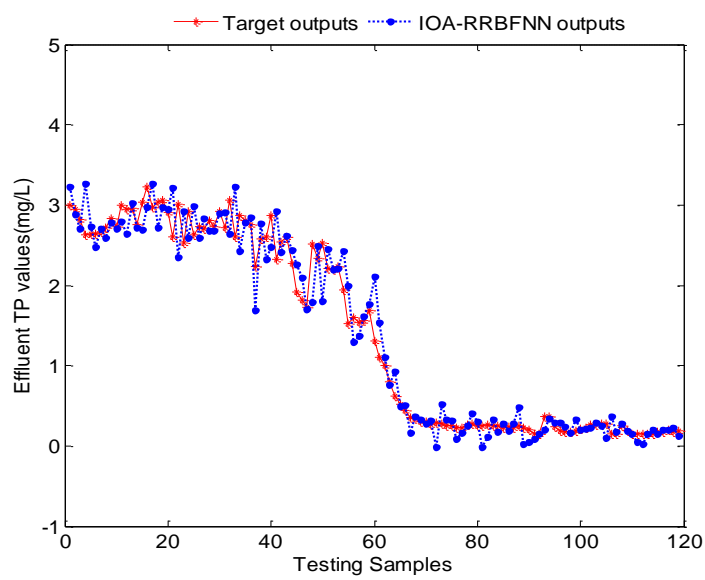

Fig. 21. The predicting results of Effluent TP by IOA-RRBFNN

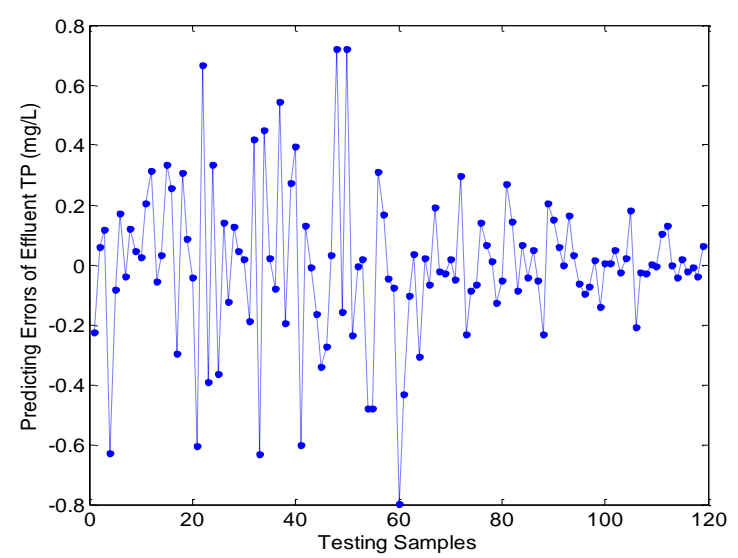

Fig. 22. The predicting errors versus testing samples

\section{V.CONCLUSION}

In this paper, an IOA-RRBFNN has been presented based on the IPSs of hidden neurons and the gradient method. Then, the performance of the proposed IOA-RRBFNN has been tested and compared with some existing approaches. The results show that the learning efficiency and performance of the algorithm is better than those of some well-known algorithms. The proposed IOA-RRBFNN owns some advantages which are summarized as follows.

1) According to the independence information between the hidden neurons and the independent component contributions from the hidden neurons to output neuron, the IPSs of hidden neurons can be calculated in the learning process. This IOA-RRBFNN introduces a more general framework for catching the information of hidden neurons, which is more objective than the other algorithms (see [22], [29]-[31]).

2) With the proposed IOA-RRBFNN, the hidden neurons can be added or pruned in the learning process, according to input and output IPS of hidden neurons. Then, the neuron splitting mechanism and deleting mechanism can mimic the dynamics of hidden neurons to choose the right active or inactive neurons. Moreover, the self-organizing mechanisms of IOA-RRBFNN are performed without presetting any thresholds, which is different from the existing methods [15]-[44].

3) It has been proven that with the structure organizing and parameters adjusting processes, the proposed IOA-RRBFNN can 
enhance the ability of nonlinear dynamic function approximation and nonlinear system prediction. In addition, the proposed

IOA-RRBFNN developed in this research yield more accurate TP predictions than the other methods in the real application.

It is worth noting that the IOA-RRBFNN is very predominant in the development of self-organizing RNN. In fact, the testing

RMSE is used to show that the IOA-RRBFNN has the advantages of compact structure and reduced errors. In the future research, the convergence of IOA-RRBFNN will be further discussed, which is crucial for the successful applications.

\section{ACKNOWLEDGMENT}

The authors also would like to thank the anonymous reviewers for their valuable comments and suggestions, which helped improve this paper greatly.

\section{REFERENCES}

[1] E. Kuriscak, P. Marsalek, J. Stroffek, P. G. Toth, Biological context of Hebb learning in artificial neural networks, a review, Neurocomputing 152 (1) (2015) 27-35.

[2] M. I. Jordanl, T. M. Mitchell, Machine learning: Trends, perspectives, and prospects, Science 349 (6245) (2015) 255-260.

[3] R. Adhikari, A neural network based linear ensemble framework for time series forecasting, Neurocomputing 157 (1) (2015) $231-242$.

[4] H.N. Nguyen, J. Zhou, H.J. Kang, A calibration method for enhancing robot accuracy through integration of an extended Kalman filter algorithm and an artificial neural network, Neurocomputing 151 (3) (2015) 996-1005.

[5] Y.C. Motai, Kernel association for classification and prediction: A survey, IEEE Transactions on neural networks and learning systems, 26 (2) (2015) 208-223.

[6] B. Goertzel, R. Lian, I. Arel, H.D. Garis, S. Chen, A world survey of artificial brain projects, Part II: Biologically inspired cognitive architectures, Neurocomputing 74 (3) (2010) 30-49.

[7] Q.H. Wu, X.J. Wang, Q.H. Shen, Research on dynamic modeling and simulation of axial-flow pumping system based on RBF neural network, Neurocomputing 186 (2016) 200-206.

[8] P. Reiner, B.M. Wilamowski, Efficient incremental construction of RBF networks using quasi-gradient method, Neurocomputing 150 (1) (2015) 349-356.

[9] H.J. Fan, Q. Song, A linear recurrent kernel online learning algorithm with sparse updates, Neural Networks 50 (2014) 142-153.

[10] B. Niu, L. Li, Adaptive neural network tracking control for a class of switched strict-feedback nonlinear systems with input delay, Neurocomputing 173 (3) (2016) 2121-2128.

[11] H.G. Han, J.F. Qiao, Adaptive computation algorithm for RBF neural network, IEEE Transactions on neural networks and learning systems 23 (2) (2012) 342-347.

[12] H.G. Zhang, Z.S. Wang, D. Liu, A comprehensive review of stability analysis of continuous-time recurrent neural networks, IEEE Transactions on neural networks and learning systems 25 (7) (2014) 1229-1262.

[13] W.I. Lee, S.Y. Lee, P. Park, Improved stability criteria for recurrent neural networks with interval time-varying delays via new Lyapunov functionals, Neurocomputing 155 (1) (2015) 128-134.

[14] H.G. Han, Y. Li, Y.N. Guo, J.F. Qiao, A soft computing method to predict sludge volume index based on a recurrent self-organizing neural network, Applied Soft Computing 38 (1) (2016) 477-486.

[15] G. Costantini, R. Perfetti, M. Todisco, Recurrent neural network for approximate nonnegative matrix factorization, Neurocomputing 138 (1) (2014) 238-247.

[16] Z.Y. Xiang, Z. Xiao, D. Wang, X.H. Li, A Gaussian mixture framework for incremental nonparametric regression with topology learning neural networks, Neurocomputing 194 (2016) 34-44.

[17] T.I. Aoki, Self-organization of a recurrent network under ongoing synaptic plasticity, Neural Networks 62 (2015) 11-19.

[18] G.B. Huang, P. Saratchandran, N. Sundararajan, An efficient sequential learning algorithm for growing and pruning RBF (GAP-RBF) networks, IEEE Trandactions on systems, man, and cybernetics-part B: cybernetics 34 (6) (2004) 2284-2292.

[19] G.B. Huang, P. Saratchandran, N. Sundararajan, A generalized growing and pruning RBF (GGAP-RBF) neural network for function approximation, IEEE Transaction on Neural Networks 16 (1) (2005) 57-67.

[20] X.B. Jia, R.Y. Wang, J.F. Liu, D.M.W. Powers, A semi-supervised online sequential extreme learning machine method, Neurocomputing 174 (2016) $168-178$.

[21] N. Vukovi, Z. Miljkovi, A growing and pruning sequential learning algorithm of hyper basis function neural network for function approximation, Neural Networks 46 (2013) 210-226.

[22] C.C. Lee, Y.C. Chiang, C.Y. Shih, C.L. Tsai, Noisy time series prediction using M-estimator based robust radial basis function neural networks with growing and pruning techniques, Expert Systems with Applications 36 (3) (2009) 4717-4724.

[23] J.P. Li, C.C. Hua, Y.G. Tang, X.P. Guan, A fast training algorithm for extreme learning machine based on matrix decomposition, Neurocomputing 173 (3) (2016) 1951-1958.

[24] H. Melo, J. Watada, Gaussian-PSO with fuzzy reasoning based on structural learning for training a Neural Network, Neurocomputing 172 (2016) $405-412$.

[25] A. Slowik, Application of an adaptive differential evolution algorithm with multiple trial vectors to artificial neural network training, IEEE Transactions on neural networks and learning systems 58 (8) (2011) 3160-3167. 
[26] J.S. Wu, J. Long, M.Z. Liu, Evolving RBF neural networks for rainfall prediction using hybrid particle swarm optimization and genetic algorithm, Neurocomputing 148 (1) (2015) 136-142.

[27] H. Chen, Y. Gong, X. Hong, Online modeling with tunable RBF network, IEEE Transactions on cybernetics 43 (3) (2013) $935-947$.

[28] C.F. Juang, Y.Y. Lin, C.C. Tu, A recurrent self-evolving fuzzy neural network with local feedbacks and its application to dynamic system processing, Fuzzy Sets and Systems 161 (19) (2010) 2552-2568.

[29] Y.Y. Lin, J.Y. Chang, C.T. Lin, Identification and prediction of dynamic systems using an interactively recurrent self-evolving fuzzy neural network, IEEE Transactions on Neural Network and learning systems 24 (2) (2013) 310-321.

[30] A. Luchetta, Automatic generation of the optimum threshold for parameter weighted pruning in multiple heterogeneous output neural networks, Neurocomputing 71 (16) (2008) 3553-3560.

[31] A. Garliauskas, Self-organized topological structures in neural networks for the visual cortex of the brain, Neurocomputing 51 (2003) 13-39.

[32] J.L. Wang, A. Belatreche, L. Maguire, T.M. McGinnity, An online supervised learning method for spiking neural networks with adaptive structure, Neurocomputing 144 (20) (2014) 526-536.

[33] X. Meng, J.F. Qiao, H.G. Han, An ART-like algorithm for constructing RBF neural networks, IEEE International Joint Conference on Neural Networks (2015) 1-7.

[34] H. Dhahri, A.M. Alimi, The modified differential evolution and the RBF (MDE-RBF) neural network for time series prediction, Neural Networks 9 (6) (2006) 2938-2948.

[35] I. Rojas, H. Pomares, J.L. Bernier, J. Ortega, B. Pino, F.J. Pelayo, A. Prieto, Time series analysis using normalized PG-RBF network with regression weights, Neurocomputing 42 (4) (2002) 267-285.

[36] C.F. Juang, A TSK-type recurrent fuzzy network for dynamic systems processing by neural network and genetic algorithms, IEEE Transactions on Fuzzy Systems, 10 (2) (2002) 155-170.

[37] X. He, T.W. Huang, C.D. Li, H.J. Che, Z.Y. Dong, A recurrent neural network for optimal real-time price in smart grid, Neurocomputing 149 (2015) $608-612$

[38] N. Subrahmanya, Y.C. Shin, Constructive training of recurrent neural networks using hybrid optimization, Neurocomputing 73 (13) (2010) $2624-2631$.

[39] C.F. Hsu, Adaptive backstepping Elman-based neural control for unknown nonlinear systems, Neurocomputing 136 (1) (2014) $170-179$.

[40] P.S. Zheng, C. Dimitrakakis, J. Triesch, Network self-organization explains the statistics and dynamics of synaptic connection strengths in cortex, Computational Biology 9 (1) (2013) 1-8.

[41] C.S. Leung, P.M. Lam, A local training-pruning approach for recurrent neural networks, International Journal of Neural Systems 13 (1) (2003) 25-38.

[42] D.T. Mirikitani, N. Nikolaev, Recursive Bayesian recurrent neural networks for time-series modeling, IEEE Transaction on Neural Networks 21 (2) (2010) $262-274$.

[43] C.F. Hsu, K. H. Cheng, Recurrent fuzzy-neural approach for nonlinear control using dynamic structure learning scheme, Neurocomputing 71 (16) (2008) 3447-3459.

[44] F.F.M. El-Sousy, Adaptive hybrid control system using a recurrent RBFN-based self-evolving fuzzy-neural-network for PMSM servo drives, Applied Soft Computing 21 (2014) 509-532.

[45] X.X. Wang, L.Y. Ma, B.S. Wang, T. Wang, A hybrid optimization-based recurrent neural network for real-time data prediction, Neurocomputing 120 (2013) 547-559.

[46] A. Woodward, T. Froese, T. Ikegami, Neural coordination can be enhanced by occasional interruption of normal firing patterns: A self-optimizing spiking neural network model, Neural Networks 62 (2015) 39-46.

[47] A. Chandrasekar, R. Rakkiyappan, Impulsive controller design for exponential synchronization of delayed stochastic memristor-based recurrent neural networks, Neurocomputing 173 (3) (2016) 1348-1355.

[48] H. Jaeger, Adaptive nonlinear system identification with echo state networks, Advances in neural information processing process (2002) 593-600.

[49] C.H. Lee, C.C. Teng, Identification and control of dynamic systems using recurrent fuzzy neural networks, IEEE Transactions on Fuzzy Systems, 8 (4) (2000) 349-366.

[50] C.J. Lin, C.C. Chin, Prediction and identification using wavelet-based recurrent fuzzy neural networks, IEEE Transactions on Systems, Man, and Cybernetics 34 (5) (2004) 2144-2154.

[51] E.Y. Cheu, H.C. Quek S.K. Ng, TNFIS: Tree-based neural fuzzy inference system, IEEE International Joint Conference on Neural Networks (2008) $398-405$.

[52] J. Kim, N. Kasabov, HyFIS: adaptive neuro-fuzzy inference systems and their application to nonlinear dynamical systems, Neural Networks 12 (9) (1999) 1301-1319.

[53] W. Li, N. Sundararajan, P. Saratchandran, A sequential learning scheme for function approximation using minimal Radial Basis Function Neural Networks, Neural Computation 9 (2) (1997) 461-478.

[54] I. Rojasa, O. Valenzuelab, F. Rojasa, A. Guillena, L.J. Herreraa, H. Pomaresa, L. Marquezb, M. Pasadas, Soft-computing techniques and ARMA model for time series prediction, Neurocomputing 71 (4) (2008) 519-537.

[55] Y.H. Chen, B. Yang, J.W. Dong, Time-series prediction using a local linear wavelet neural network, Neurocomputing 69 (6) (2006) 449-465.

[56] S. Wu, M.J. Er, Y. Gao, A fast approach for automatic generation of fuzzy rules by generalized dynamic fuzzy neural networks IEEE Transactions Fuzzy System 9 (4) (2001) 578-594.

[57] S. Yilmaz, Y. Oysal, Fuzzy wavelet neural network models for prediction and identification of dynamical systems, IEEE Transactions on Neural Network 21 (10) (2010) 1599-1609. This work was supported by the National Science Foundation of China under Grants 61203099, 61533002 and 61225016, China Postdoctoral Science Foundation under Grant 2014M550017, Ph.D. Program Foundation from Ministry of Chinese Education under Grant 20131103110016, and Beijing Municipal Education Commission Foundation under Grants km201410005001 and KZ201410005002. Asterisk indicates corresponding author. 